\title{
FGF-23 in chronic kidney disease
}

\author{
Volume 4 Issue 6 - 2017
}

\section{Letter to Editor}

Halankar et al. ${ }^{1}$ have reported significant high levels of intact FGF-23(iFGF-23) (19.0 fold, $\mathrm{p}=0.001)$ and tremendous increase in C-terminal FGF-23 (C-terminal FGF-23) (49.7 fold, $\mathrm{p}=0.001$ ) in the circulation of CKD- stage 5 (dialytic CKD) patients as compared to age and gender matched controls which did not decrease at six month on regular treatment of Calcium, Calcitriol and Phosphate binders. At recruitment, significant correlation between $\mathrm{P}$ and iFGF-23 (Spearman (rs) $=0.317, \mathrm{p}=0.028$ ), was also observed. The above mentioned increase in the levels of C-terminal FGF-23 have been suggested due to reduced renal clearance causing marked accumulation of C-terminal fragments in chronic hemodialysis patients ${ }^{2}$ which in agreement with Goetz et al. ${ }^{3}$ may interfere in the phosphaturic activity of the active iFGF-23 causing significant increase in P levels at the dialytic stage of $\mathrm{CKD}$ patients. Apart from the reduced renal clearance and accumulation of C-terminal fragments, ${ }^{2}$ other supposition suggested is, the increasing stimuli for FGF-23 production with deteriorating renal function could possibly overwhelm the capacity of the physiological inactivation mechanism that ordinarily controls the bioactive FGF-23 concentrations. It has been reported that increased production of FGF-23 by the bone may contribute to elevated levels in CKD and ESRD. ${ }^{4,5}$ However, the biologic activity of C-terminal FGF-23 fragments remains a controversial topic and the mechanism postulated to explain the reduced renal clearance or decreased degradation rate of $\mathrm{C}$ terminal FGF-23 in CKD remains unclear.

Apart from the above findings, the authors would like to further add that in CKD stage 5 patients at six month, an inverse correlation between iFGF-23 and C-terminal FGF-23 ( $r s=-0.357, p=0.013$ ) has also been observed. This indicates that the levels of iFGF-23 decreased with significant increased C-terminal FGF-23 levels. In order to transmit its signal, full length mature FGF-23 forms a heterotrimer complex with receptor FGF1Rc and co-receptor Klotho. Klotho binds to the C-terminal of FGF-23. Between the N-terminal and C-terminal domains of FGF-23 there is a cleavage site at 179 amino acid (amino acid number counting from signal peptide) that contains an RXXR motif which is the cleavage site for enzymes of the proconvertasetype of subtilisin or kexine type of the serine protease family. In its dynamics, active iFGF-23 is proteolytically cleaved at its C-terminal to produce inactive $\mathrm{C}$-terminal and $\mathrm{N}$ - terminal fragments. ${ }^{6,7}$ Thus, C-terminal FGF-23 is produced by proteolytic cleavage of active iFGF-23 during functional dynamics of FGF-23.

In dialytic CKD patients, the direct correlation between $\mathrm{P}$ and iFGF-23 at recruitment suggests that at elevated $P$, stimulus is towards production of active iFGF-23 to inhibit the NaPi-2a and $\mathrm{NaPi}-2 \mathrm{c}$ co-transporters and prevent further absorption of $\mathrm{P}$. The inverse correlation observed between iFGF-23 and C-terminal FGF-23 levels at six month suggests that although initial stimuli of elevated $\mathrm{P}$ is to increase the iFGF-23, there is increase in C-terminal FGF-23 levels at six month and this increase may be from some other source then cleavage of C-terminal FGF-23 fragments from iFGF-23 during its functional dynamics. As at CKD stage 5, the nephron mass is less

\author{
Kavita K Shalia, ${ }^{3}$ Sandhya Sivaraman, ${ }^{2}$ Arun \\ Halankar' \\ 'Department of Nephrology, Sir H N Reliance Foundation \\ Hospital and Research Centre, India \\ ${ }^{2}$ Ph.D. Student, Sir H N Hospital and Research Centre, India \\ ${ }^{3} \mathrm{Sr}$ Scientist, Sir H N Medical Research Society, India
}

Correspondence: Kavita Shalia, Sr Scientist, Sir H N Medical Research Society, Court House, LT Road, Mumbai 400002 , Maharashtra, India, Tel 022 67673883, Fax 022 67673898, Email Kavita.shalia@rfhospital.org

Received: May 31, 2017| Published: June 09, 2017

and so is $\alpha$-Klotho, co-receptor for FGF-23, to carry out its function. Decrease in soluble Klotho levels ${ }^{7}$ reflect non-availability of $\alpha$-Klotho co-receptor for FGF-23 in kidney which leads to reduction in inhibition of NaPi co-transporters. The increase in P levels (hyperphosphatemia) due to only absorption and no excretion have stimulated increased FGF-23 levels and more so of C-terminal FGF-23. At elevated P level in dialytic CKD stage, elevation in C-terminal FGF-23 levels suggests some defects at FGF-23 expression, translational or post translational, that may cause an excess production or secretion of only C-terminal FGF-23 fragments over iFGF-23. These further dues to reduced clearance accumulate and subsequently inhibit the activity of iFGF23 and not allow the control of hyperphosphatemia.

In an another study from our group on pre-dialytic CKD patients (CKD stage 1-4), ${ }^{8}$ an inverse correlation between $\mathrm{P}$ and eGFR ( $($ Pearson $(\mathrm{r})=-0.467, \mathrm{p}=0.021)$, direct correlation between $\mathrm{P}$ and C-terminal FGF-23 ( $\mathrm{rs}=-0.464, \mathrm{p}=0.022$ ) and tremendous increase in C terminal FGF-23 levels (CKD stage 1,2- 19.2\%, $\mathrm{p}=0.001$, CKD stage $3,4-32.3 \%, p=0.001$ ) as compared to age, gender and BMI matched controls have been observed. This suggests that although within normal range, $P$ levels increased with decrease in eGFR and up-regulated the FGF-23 levels. At CKD stage 1-4, at moderate to mild Kidney function and availability of $\alpha$-Klotho, ${ }^{7}$ would take care of excretion of $\mathrm{P}$, in spite of presence of C-terminal FGF-23 fragments. At six month, there was direct correlation observed between iFGF-23 and C-terminal FGF-23 ( $r s=0.543, p=0.007)$ (additional result). However, the fold increase of C-terminal FGF-23 above iFGF-23 (CKD stage 1, $2-41.8$ fold and CKD stage 3, $4-60.6$ fold) and correlation between $\mathrm{P}$ and C-terminal FGF-23 at recruitment, again suggests an additional source of only C-terminal FGF-23, independent from functional dynamics of co-transport inhibitory mechanism, which needs to be identified.

\section{Acknowledgments}

None. 


\section{Conflicts of Interset}

None.

\section{References}

1. Halankar A, Sivaraman S, Shalia K. FGF-23 and Hyperphosphatemia in Dialysis Dependent Chronic Kidney Disease Patients. UrolNephrol Open Access J. 2017;4(5):00141.

2. Weber TJ, Liu S, Indridason OS, et al. Serum FGF23 levels in normal and disordered phosphorus homeostasis. J Bone Miner Res. 2003;18(7):1227-1234.

3. Goetz R, Nakada Y, Hu MC, et al. Isolated C-terminal tail of FGF23 alleviates hypophosphatemia by inhibiting FGF-23-FGFR-Klotho complex formation. Proc Natl Acad Sci U S A. 2010;107(1):407-412.
4. Pereira RC, Jüppner H, Azucena-Serrano CE, et al. Patterns of FGF-23, DMP1, and MEPE expression in patients with chronic kidney disease. Bone. 2009;45(6):1161-1168.

5. Kurosu H, Ogawa Y, Miyoshi M, et al. Regulation of fibroblast growth factor-23 signalling by Klotho. J Biol Chem . 2006;281(10):6120-6123.

6. Shimada T, Muto T, Urakawa I, et al. Mutant FGF-23 responsible for autosomal dominant hypophosphatemic rickets is resistant to proteolytic cleavage and causes hypophosphatemia in vivo. Endocrinology. 2002;143(8):3179-3182.

7. Sivaraman S, Halankar A, Shalia K. Klotho in chronic kidney disease in Indian population. Inter J of Curr Res. 2006;8(11):42477-42485.

8. Sivaraman S, Halankar A, Shalia K. Fibroblast Growth Factor (FGF-23) in Pre-Dialytic Chronic Kidney Disease Patients. Inter J of Biomed Res. 2016;7(9):663-670. 